\title{
Explicit Time Stepping Methods with High Stage Order and Monotonicity Properties
}

\author{
Emil Constantinescu ${ }^{1}$ and Adrian Sandu ${ }^{2}$ \\ 1 Mathematics and Computer Science Division, \\ Argonne National Laboratory, Argonne, IL 60439, USA \\ 2 Department of Computer Science, Virginia Tech, Blacksburg, VA 24061, USA
}

\begin{abstract}
This paper introduces a three and a four order explicit time stepping method. These methods have high stage order and favorable monotonicity properties. The proposed methods are based on multistagemultistep (MM) schemes that belong to the broader class of general linear methods, which are generalizations of both Runge-Kutta and linear multistep methods. Methods with high stage order alleviate the order reduction occurring in explicit multistage methods due to non-homogeneous boundary/source terms. Furthermore, the MM schemes presented in this paper can be expressed as convex combinations of Euler steps. Consequently, they have the same monotonicity properties as the forward Euler method. This property makes these schemes well suited for problems with discontinuous solutions.
\end{abstract}

\section{Introduction}

The numerical solution of time-dependent partial differential equations and nonlinear hyperbolic conservation laws are of great practical importance as they model diverse physical phenomena that appear in engineering, aeronautics, astrophysics, meteorology oceanography, environmental sciences, etc. Representative examples for nonlinear hyperbolic conservation laws include gas dynamics, shallow water flow, ground-water flow, non-Newtonian flows, traffic flows, advection and dispersion of contaminants, etc.

In the "method of lines" approach the temporal and spatial discretizations are independent. Traditionally Runge-Kutta (RK) and linear multistep methods (LM)s have been used for the integration of ODEs and semi-discrete timedependent PDEs. General linear (GL) methods [12] represent a natural generalization of both Runge-Kutta $(\mathrm{RK})$ and linear multistep (LM) methods. The methods investigated in this work are based on multistage-multistep (MM) schemes that belong to the broader class of GL methods. Multistep-multistage schemes are aimed at enhancing the stability and accuracy properties of the classical RK and LM methods. They use both internal stages like RK methods and information from previous solution steps like LM methods.

Explicit Runge-Kutta methods have stage order equal to one, and hence are subject to order reduction in the presence of non-homogeneous boundary and source terms 31314. The proposed high-stage order MM methods alleviate

G. Allen et al. (Eds.): ICCS 2009, Part II, LNCS 5545, pp. 293-301, 2009.

(C) Springer-Verlag Berlin Heidelberg 2009 
this problem without loosing their explicit character. Moreover, they can be expressed as convex combinations of Euler steps, and consequently, they have the same monotonicity properties that the spatial discretization method has with the forward Euler time stepping scheme, but with a different time step restriction 156]. This property makes the proposed MM schemes well suited for problems with discontinuous solutions (e.g., hyperbolic problems). Furthermore, the monotonicity properties can also guarantee the positivity of the solution.

In this study we investigate explicit time stepping methods of orders three and four based on MM schemes. The proposed methods have high stage order and favorable monotonicity properties. These features allow them to:

- avoid order reduction due to non-homogeneous boundary/source terms and

- prevent non-physical behavior with discontinuous solutions.

The proposed methods are aimed at modeling the transport components in atmospheric and oceanic simulations. The rest of this manuscript is organized as follows. In Sections 2 and 3 we present some background material on MM schemes and the monotonicity property considered in this study. The two proposed methods are presented in Sec. 4. Numerical experiments that illustrate the main features of the new methods are shown in Sec. 5. A short discussion concludes the paper.

\section{Problem Formulation and Monotonicity Considerations}

In this work we are concerned with the numerical solution of nonlinear timedependent partial differential equations in the method of lines approach:

$$
y^{\prime}(t)=f(t, y(t)), t_{0}<t<t_{\text {Final }}, y\left(t_{0}\right)=y_{0},
$$

where $f$ represents the discretization of the spatial variables forming a semidiscrete equation, continuous in time. System (1) is nonauthonomous, however, for brevity we skip the time argument of $f$, unless noted otherwise.

We next introduce the concept of strong stability which defines the monotonicity properties that the proposed methods obey.

Definition 1 (Strong stability 12,6,15]). A sequence $\left\{y^{(n)}\right\}$ is said to be strongly stable in a given semi-norm $\|\cdot\|$ if $\left\|y^{(n+1)}\right\| \leq\left\|y^{(n)}\right\|$ for all $n \geq 0$.

Strong stability preserving (SSP) integrators are high order time stepping schemes that preserve the stability properties of the spatial discretization used with explicit Euler time stepping. Spurious oscillations can occur in a numerical solution that obeys the classical linear stability [6]. In PDEs with hyperbolic components an appropriate spatial discretization combined with an SSP time stepping method yields a numerical solution that does not exhibit nonlinear instabilities.

The favorable properties of SSP schemes derive from convexity arguments. In particular, if the forward Euler method is strongly stable for any time step smaller than $\Delta t_{\mathrm{FE}}$ (i.e., $\|y+\Delta t f(y)\| \leq\|y\|, \Delta t \leq \Delta t_{\mathrm{FE}}$ ), then higher-order 
methods can be constructed as convex combinations of forward Euler steps with various step sizes [15]. For example an explicit $s$-stage Runge-Kutta method can be represented in Euler steps:

$$
\begin{aligned}
& y_{[n]}=y_{[n-1]}^{(s+1)}, \quad y_{[n-1]}^{(1)}=y_{[n-1]}, \\
& y_{[n-1]}^{(i)}=\sum_{j=1}^{i-1}\left[\alpha^{(i, j)} y_{[n-1]}^{(j)}+\beta^{(i, j)} \Delta t F_{[n-1]}^{(j)}\right] ; i=2,3, \ldots, s, s+1 .
\end{aligned}
$$

SSP methods preserve the strong stability of the forward Euler scheme for bounded time steps $\Delta t \leq \mathcal{C} \cdot \Delta t_{\mathrm{FE}}$, where $\mathcal{C}$ is referred to as the CFL coefficient for the SSP property.

Theorem 1 (SSP for Runge-Kutta methods 6 15]). If the forward Euler method is strongly stable under the CFL restriction $\Delta t \leq \Delta t_{\mathrm{FE}}$, then the RungeKutta method (2) with $\beta^{(i, j)} \geq 0$ is $S S P$ provided that $\Delta t \leq \mathcal{C} \Delta t_{\mathrm{FE}}$, where

$$
\mathcal{C}=\operatorname{Min}\left\{\left(\alpha^{(\mathrm{i}, \mathrm{j})} / \beta^{(\mathrm{i}, \mathrm{j})}\right): 1 \leq \mathrm{i} \leq \mathrm{s}, 1 \leq \mathrm{j} \leq \mathrm{i}-1, \beta^{(\mathrm{i}, \mathrm{j})} \neq 0\right\}
$$

In order to compare methods with different computational cost, a scaled or effective CFL coefficient, denoted by $\widehat{\mathcal{C}}$, is obtained by scaling the method's CFL with the number of right-hand-side evaluations.

\section{Multistep Multistage Methods}

We consider the following explicit $k$-step $s$-stage multistep-multistage method to compute the numerical solution of (1) with time step $\Delta t$. The solution at step $n, y_{[n]} \approx y\left(t_{n}\right)=y(n \Delta t)$ is given by

$$
\begin{aligned}
y_{[n]}= & y_{[n-1]}^{(s+1)}, \quad y_{[n-1]}^{(1)}=y_{[n-1]}, \\
y_{[n-1]}^{(i)} & =\sum_{\ell=2}^{k} \sum_{j=1}^{s}\left[\alpha_{[n-\ell]}^{(i, j)} y_{[n-\ell]}^{(j)}+\beta_{[n-\ell]}^{(i, j)} \Delta t F_{[n-\ell]}^{(j)}\right]+ \\
& +\sum_{j=1}^{i-1}\left[\alpha_{[n-1]}^{(i, j)} y_{[n-1]}^{(j)}+\beta_{[n-1]}^{(i, j)} \Delta t F_{[n-1]}^{(j)}\right] ; i=2,3, \ldots, s, s+1,
\end{aligned}
$$

where $F_{[n-\ell]}^{(i)}=f\left(t_{[n-\ell]}+c_{i} \Delta t, y_{[n-\ell]}^{(i)}\right)$. We refer to $y_{[n-\ell]}^{(i)}, i=1 \ldots s+1, \ell=$ $1 \ldots k$ as the stage $i$ value at step $n-\ell$, and to $F_{[n-\ell]}^{(i)}$ as the corresponding stage derivative. The first sum in (3b) represents linear combinations of stage values and derivatives evaluated at previous steps, whereas the second sum describes the internal stages of the current step evaluation. Each stage value $y_{[n-\ell]}^{(i)}$ is an approximation to $y\left(t_{n-\ell}+c_{i} \Delta t\right)$. The abscissa, $c$, is determined from the consistency conditions. 
The linear stability of method (3) is analyzed on a linear scalar test problem: $y^{\prime}(t)=\lambda y(t), \lambda \in \mathbb{C}$. By applying (3) to the test problem yields a solution of form $y^{n+1}=R(z) y^{n}$, where $z=\lambda \Delta t$ and $R(z)$ is referred to as the stability function of the method. Method (3) is linearly stable if $|R(z)| \leq 1$. The linear stability region is defined as the set $\mathcal{S}=\{z \in \mathbb{C}:|R(z)| \leq 1\}$.

We give the following result without proof.

Theorem 2 (SSP for MM methods). If the forward Euler method is strongly stable under the CFL restriction $\Delta t \leq \Delta t_{\mathrm{FE}}$, then method (3) with $\beta_{[n-\ell]}^{(i, j)} \geq 0$ is $S S P$ provided that $\Delta t \leq \mathcal{C} \Delta t_{\mathrm{FE}}$, where

$$
\mathcal{C}=\operatorname{Min}\left\{\left(\alpha_{[\mathrm{n}-\ell]}^{(\mathrm{i}, \mathrm{j})} / \beta_{[\mathrm{n}-\ell]}^{(\mathrm{i}, \mathrm{j})}\right): 1 \leq \mathrm{i} \leq \mathrm{s}, 1 \leq \mathrm{j} \leq \mathrm{i}-1,1 \leq \ell \leq \mathrm{k}, \beta_{[\mathrm{n}-\ell]}^{(\mathrm{i}, \mathrm{j})} \neq 0\right\} .
$$

By using consistency and convexity arguments the above theorem reduces to Theorem 1 and the proof is given in 6]. The importance of the SSP property is illustrated in Fig. 2, a where non-physical oscillations develop in the solution.

\section{The Proposed Methods}

In this section we present two new explicit multistep-multistage schemes that have stage order equal to three and are strong stability preserving.

\subsection{Method MM p3 q3}

Method MM p3 q3 (4) is an order three and stage order three $(p=3, q=3)$ MM method with three stages and two steps $(s=3, k=2)$. The CFL coefficient is $\mathcal{C}=1.44(\widehat{\mathcal{C}}=0.48)$.

$$
\begin{aligned}
& \alpha_{[n-1]}^{(2,1)}=0.697169114587643 \beta_{[n-1]}^{(2,1)}=0.484471495618137 \\
& \alpha_{[n-1]}^{(3,2)}=0.76354468478889 \beta_{[n-1]}^{(3,2)}=0.530596705549337 \\
& \frac{\alpha_{[n-1]}^{(4,3)}=0.816170594740032 \beta_{[n-1]}^{(4,3)}=0.567167105426239}{\alpha_{[n-2]}^{(2,1)}=0.302830885412357 \beta_{[n-2]}^{(2,1)}=0.109139040169882} \\
& \alpha_{[n-2]}^{(3,1)}=0.23645531521111 \beta_{[n-2]}^{(3,1)}=0.109233120743169 \\
& \frac{\alpha_{[n-2]}^{(4,1)}=0.183829405259968 \beta_{[n-2]}^{(4,1)}=0.106231031926622}{c=[0,0.290779650375662,0.625397767570505,1]^{T} .}
\end{aligned}
$$

\subsection{Method MM p4 q3}

Method MM p4 q3 (5) is an order four and stage order three $(p=4, q=3) \mathrm{MM}$ method with two stages and four steps $(s=2, k=4)$. The CFL coefficient is $\mathcal{C}=0.64(\widehat{\mathcal{C}}=0.32)$. 


$$
\begin{aligned}
& \alpha_{[n-1]}^{(2,1)}=0.641788036235959 \beta_{[n-1]}^{(2,1)}=1 . \\
& \frac{\alpha_{[n-1]}^{(3,2)}=0.530533524263627 \beta_{[n-1]}^{(3,2)}=0.826649133840462}{} \\
& \frac{\alpha_{[n-2]}^{(3,1)}=0.278475821635639 \beta_{[n-2]}^{(3,1)}=0.433906221232917}{\alpha_{[n-3]}^{(2,1)}=0.295361832953222 \beta_{[n-3]}^{(2,1)}=0.354153138170544} \\
& \frac{\alpha_{[n-3]}^{(3,1)}=0.111760513607703 \beta_{[n-3]}^{(3,1)}=0.174139291008244}{\alpha_{[n-4]}^{(2,1)}=0.062850130810818} \\
& \frac{\alpha_{[n-4]}^{(3,1)}=0.07923014049303}{c=[0,0.574879079831644,1]^{T} .}
\end{aligned}
$$

\subsection{Linear Stability}

The linear stability region for MM p3 q3 (4) is shown in Figure 1)a. We remark that the stability region contains a segment of the imaginary axis, which is a desirable property when solving PDEs via the method of lines with certain spatial discretizations 9 .

In Figure 1 b we show the stability region of MM p4 q3 (5), and here we note again that the stability region contains a segment of the imaginary axis. The region is smaller than in the case of (4); however, the fourth order method requires only two function evaluation. It follows that MM p4 q3 has two thirds of the cost of MM p3 q3.

\subsection{Starting Procedures}

Each step of the MM method (3) requires past precomputed information, specifically, $y_{[n-\ell]}^{(j)}$ and $F_{[n-\ell]}^{(j)}, 2 \leq \ell \leq k$. In this study the initial step is considered to provide an approximation to the exact solution and its derivative at the corresponding time within order $p$, the order of the MM method under consideration.

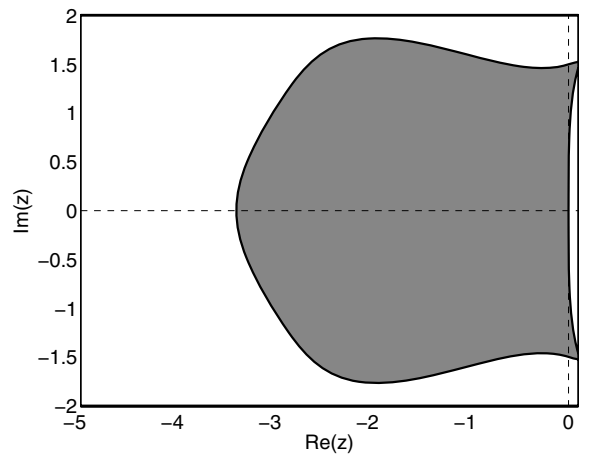

a)MM p3 q3

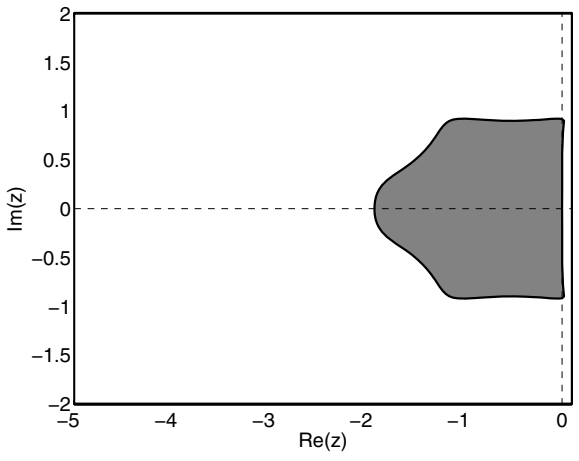

b) MM p4 q3

Fig. 1. Linear stability regions (shaded) for MM p3 q3 and MM p4 q3 
In practice, for the proposed methods, one can easily compute the initial solution and its derivative components with the classical SSP RK schemes 68 of corresponding orders and at the respective times as given by the method abscissa.

\section{$5 \quad$ Numerical Results}

In this section we present two numerical experiments that illustrate the properties of the two proposed MM methods. In the first experiment we investigate the SSP (monotonicity) properties. In the second numerical experiment we present the order reduction phenomenon, and show how it can degrade the accuracy of high order $(p)$ low stage order $(q=1)$ multistage methods. We further show that the proposed methods maintain their corresponding orders of consistency $(p)$.

\subsection{Monotonicity}

Methods with SSP properties are needed to evolve in time solutions that may develop discontinuities of hyperbolic PDEs. The SSP conditions impose a very strict restriction on the time steps, and hence the time stepping scheme efficiency is very important.

Figure 2 shows the solutions of the advection equation obtained with the proposed methods MM p3 q3 (4) and MM p4 q3 (5) and the optimal third order RK scheme, RK3, with three stages, $\mathcal{C}=1(\widehat{\mathcal{C}}=0.33)$ 68 . The space discretization is first order upwind, chosen for its well understood behavior. The time step for MM p3 q3 is such that the CFL coefficient is 1.3 . At $t=0.22$ (Figure 2,a) the MM method solution remains oscillation free, while the RK3 solution shows the effects of linear instability. The solution obtained by using MM p4 q3 (Figure2.b) is also stable, but at a lower CFL coefficient comparable, however, with the one used for the MM p3 q3 case; i.e., MM p3 q3 and RK3 require three function evaluations per step, and hence, a CFL of two thirds is needed for a fair comparison.

We next explore the monotonicity properties of the SSP MM methods on a nonlinear hyperbolic equation. The inviscid Burgers' equation is

$$
\frac{\partial y(t, x)}{\partial t}+\frac{\partial}{\partial x}\left(\frac{1}{2} y(t, x)^{2}\right)=0, \quad 0 \leq x \leq 1,0 \leq t \leq t_{\text {Final }} .
$$

The spatial discretization uses the third-order upwind-biased flux limited scheme based 41011. This spatial discretization is SSP with forward Euler steps and hence, with the proposed MM methods described in this work. The SSP condition is satisfied if the CFL coefficient of the method $\mathcal{C}$ is smaller than the CFL number of the problem: $\mathcal{C} \leq$ problem $\mathrm{CFL}$ number $=\max (y) \Delta t / \Delta x$.

In Figure 3 we show the solution of the Burgers' equation integrated with RK3 $(s=3, \mathcal{C}=1)$ and MM p3 q3 (4) $(\mathcal{C}=1.44)$ at time 0.25 with a method CFL of 1.5 . 


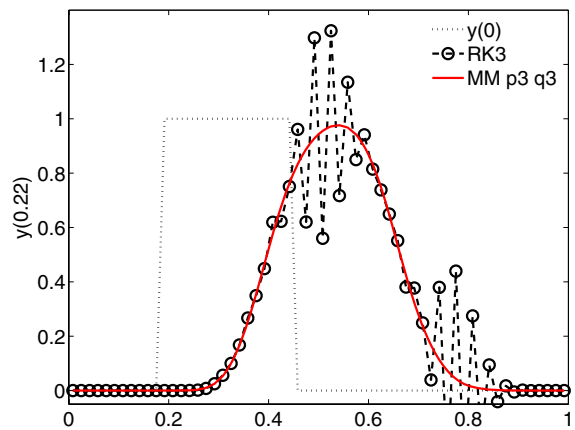

a) $\mathrm{MM} \mathrm{p} 3 \mathrm{q} 3, \mathcal{C}=1.3$

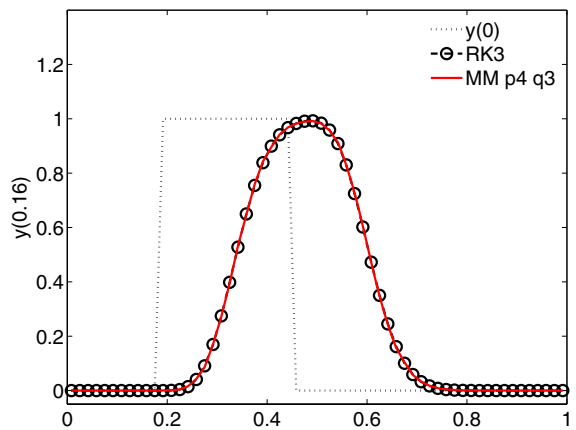

b) $\mathrm{MM} \mathrm{p} 4 \mathrm{q} 3, \mathcal{C}=0.86$

Fig. 2. Solution of the advection equation integrated in time with RK3 and the multistep-multistage schemes MM p3 q3 and MM p4 q3

The solution given by the MM scheme remains oscillation free, whereas the classical RK method becomes unstable.

\subsection{Avoiding Order Reduction}

Order reduction describes the behavior where the effective order of a numerical method on a given problem is smaller than its theoretical order as given by the classical theory. Order reduction can considerably degrade the efficiency of the numerical integration. Moreover, order reduction is difficult to detect in practical computations because embedded methods used for error estimation are also affected by it.

Explicit RK methods have the stage order equal to one, which makes them susceptible of order reduction for problems with non-homogeneous boundary conditions and/or nonzero source terms. In order to illustrate this, we consider the test problem from 14 (advection with a nonlinear source

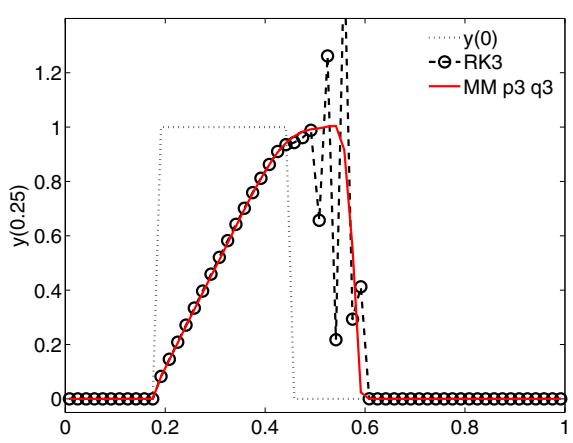

Fig. 3. Solution of Burgers' equation integrated in time with RK3 $(\mathcal{C}=1.00)$ and the multistep-multistage schemes MM p3 q3 $(\mathcal{C}=1.44)$. The CFL of the problem is 1.5 . term):

$$
\frac{\partial y(t, x)}{\partial t}=-\frac{\partial y(t, x)}{\partial x}+b(t, x), \begin{aligned}
& 0 \leq x \leq 1 \\
& 0 \leq t \leq 1
\end{aligned}, \begin{aligned}
& y(t, 0)=b(t, 0) \\
& y(0, x)=y_{0}(x)
\end{aligned}
$$

The initial condition is $y_{0}(x)=1+x$ and the (left) boundary and source term is $b(t, x)=(t-x) /(1+t)^{2}$. The exact solution given by $y(t, x)=(1+x) /(1+t)$ is 


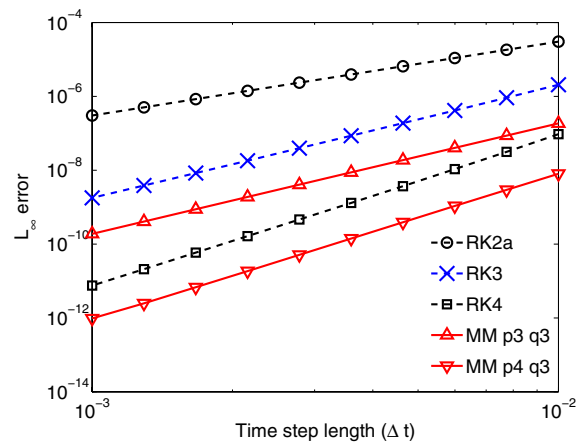

a) Error vs. time step - no forcing

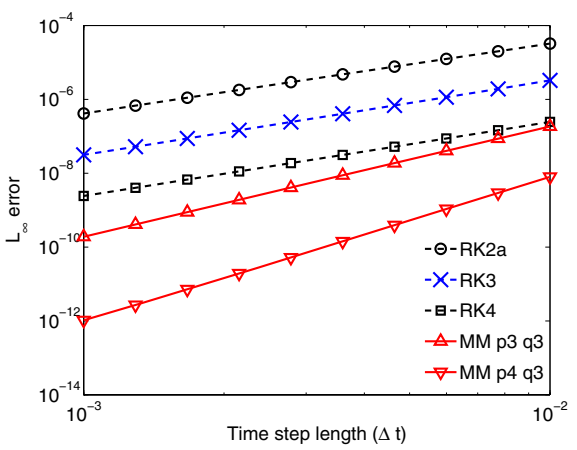

b) Error vs. time step - with forcing

Fig. 4. Numerical illustration of the order reduction phenomenon. The $L_{\infty}$ norm of error is shown versus the time step length for RK methods of orders 2-4 and MM p3 q3 and MM p4 q3. (a) Results with no forcing show that the effective order of each method equals its theoretical order. (b) When forcing is present the effective order of RK methods is two (order reduction). The high stage-order MM methods maintain their theoretical orders of accuracy.

linear in space, allowing us to use first order upwind space discretization without introducing discretization errors. For the time integration we employ the typical RK methods of orders 2, 3, and 4. Sanz-Serna et al. [14] show that RK methods with $p \geq 3$ suffer from order reduction. This theoretical result is verified in our numerical experiment.

Figure 4, a shows the discretization error versus the time step with the forcing terms switched off [14. In this case all methods retain their expected order, verifying the classical theory. In Figure 4, b we show the results with stiff boundary and source terms. In this case both the third order RK3a method [5] and the fourth order "classical" RK4 method 7] display second order behavior. In these situations a second order method can be more efficient than higher order methods. The high stage order proposed methods MM p3 q3 (4) and MM p4 q3 (5) retain their corresponding orders of consistency.

\section{Discussion}

In this paper we introduce two new explicit multistage-multistep methods with high stage orders for solving ordinary differential equations and PDEs via the method of lines. The MM methods are SSP - they have the monotonicity properties of forward Euler scheme, but under a different time step restriction.

To our knowledge the proposed methods are the first explicit high-stage order SSP methods. The two numerical experiments presented in this paper motivate both properties - SSP and high stage order.

An error control mechanism can be considered by using a lower order embedded method; however, changing the time step requires restarting the method. 


\section{Acknowledgement}

This work was supported by the Office of Advanced Scientific Computing Research, Office of Science, U.S. Department of Energy, under Contract DE-AC0206CH11357.

\section{References}

1. Burrage, K., Butcher, J.C.: Non-linear stability of a general class of differential equation methods. BIT 20(2), 185-203 (1980)

2. Butcher, J.C.: General linear methods for ordinary differential equations. Mathematics and Computers in Simulation (2007) (in press)

3. Carpenter, M.H., Gottlieb, D., Abarbanel, S., Don, W.-S.: The theoretical accuracy of Runge-Kutta time discretizations for the initial boundary value problem: A study of the boundary error. SIAM Journal on Scientific Computing 16(6), 1241$1252(1995)$

4. Chakravarthy, S., Osher, S.: Numerical experiments with the Osher upwind scheme for the Euler equations. AIAA Journal 21, 1241-1248 (1983)

5. Gottlieb, S.: On high order strong stability preserving Runge-Kutta and multi step time discretizations. Journal of Scientific Computing 25(1), 105-128 (2005)

6. Gottlieb, S., Shu, C.-W., Tadmor, E.: Strong stability-preserving high-order time discretization methods. SIAM Rev. 43(1), 89-112 (2001)

7. Hairer, E., Norsett, S.P., Wanner, G.: Solving Ordinary Differential Equations II: Stiff and Differential-Algebraic Problems. Springer, Heidelberg (1993)

8. Higueras, I.: On strong stability preserving time discretization methods. Journal of Scientific Computing 21(2), 193-223 (2004)

9. Hundsdorfer, W., Verwer, J.G.: Numerical Solution of Time-Dependent AdvectionDiffusion-Reaction Equations, vol. 33. Springer, Heidelberg (2003)

10. Osher, S., Chakravarthy, S.: High resolution schemes and the entropy condition. SIAM Journal on Numerical Analysis 21(5), 955-984 (1984)

11. Osher, S., Chakravarthy, S.: Very high order accurate TVD schemes. Oscillation Theory, Computation, and Methods of Compensated Compactness, IMA Vol. Math. Appl. 2, 229-274 (1986)

12. Ruuth, S.J., Hundsdorfer, W.: High-order linear multistep methods with general monotonicity and boundedness properties. Journal of Computational Physics 209(1), 226-248 (2005)

13. Sanz-Serna, J.M., Verwer, J.G.: Stability and convergence at the PDE/stiff ODE interface. Appl. Numer. Math. 5(1-2), 117-132 (1989)

14. Sanz-Serna, J.M., Verwer, J.G., Hundsdorfer, W.: Convergence and order reduction of Runge-Kutta schemes applied to evolutionary problems in partial differential equations. Numer. Math. 50(4), 405-418 (1987)

15. Shu, C.-W., Osher, S.: Efficient implementation of essentially non-oscillatory shockcapturing schemes. Journal of Computational Physics 77(2), 439-471 (1988) 